

\section{DISCLAIMER}

This report was prepared as an account of work sponsored by an agency of the United States Government. Neither the United States Goverament nor any agency thereof, nor any of their employees, makes any warranty. express or implied, or assumes any legal liability or responsibility for the accuracy, completeness, or usefulness of any information, apparatus, product, or process disclosed, or represents that its use would not infringe privately owned rights. Reference herein to any specific commercial product, process, or service by trade name, tradernark, manufacturer, or otherwise does not necessarily constitute or imply its endorsement, recommendation, or favoring by the United States Government or any agency thereof. The views and opinions of authors expressed herein do not nocessarily state or reflect those of the United States Government or any agency thereof. 


\title{
THE DIII-D SUMMARY CONTRIBUTION TO THE FINAL INTOR WORKSHOP
}

\author{
D.N. Hill, S.L. Allen, N.H. Brooks, ${ }^{a}$ D. Content, ${ }^{b}$ J.C. DeBoo, \\ P. Gohil, G. HaAs, ${ }^{c}$ D. Heifetz, ${ }^{d}$ C.L. Hsieh, ${ }^{a}$ R.A. Hulse, ${ }^{d}$

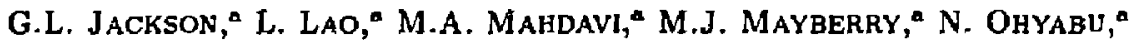 \\ D.E. Perkins, M.E. Perry, ${ }^{b}$ T.W. Petrie, M.E. Rensink, \\ T. Scoville," M. Shimada, " R.T. SNider," R.E. Stockdale," and R. Wood
}

\section{Introduction and Summary}

Impurities must be controlled in high temperature tokamak plasmas because the impurity radiation can limit the plasma temperature to values below that required for ignition. Impurity influx can be reduced with the use of the magnetic divertor configuration, which surrounds the hot dense plasma with a much cooler plasma that contacts material walls only on open field lines. Many present day tokamaks, such as ASDEX, DIII-D, JET, and JFT-2M routinely operate with divertor plasmas, and as a result have obtained the $\mathrm{H}$-mode with its good energy confinement.

In DIII-D, good $\mathrm{H}$-mode confinement $\left[\tau_{\mathrm{E}}(\mathrm{NBI})=\tau_{\mathrm{E}}(\mathrm{OH}) \simeq 120-150 \mathrm{~ms}\right.$ at $\left.I_{p}=1 \mathrm{MA}\right]$ has been obtained $[1,2]$ with up to $6 \mathrm{MW}$ of neutral-beam injection (NBI). In this report vie summarize the essential features of divertor operation for DIII-D H-mode plasmus. Briefly, our measurements show that:

(1) Large edge density and temperature gradients are obtained with scale lengths of a few $\mathrm{cm}$ at the midplane.

(2) In the steady state, approximately $10-20 \%$ of the input power is deposited on the divertor plates in a region that is peaked on the separatrix and extends radially over $\simeq 2-4 \mathrm{~cm}$ at the outboard intercept. More power (2:1) reaches the outboard intercept than the inboard.

\footnotetext{
- GA Technologies Inc.

b Johns Hopkins University

c Max-Planck-Instit ut für Plasmaphysik

d Princeton Plasma Physics Laboratory

- Japan Atomic Energy Research Institute
}

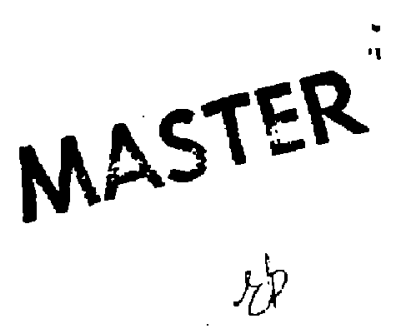


(3) Low electron temperature $\left(\leq 20 \mathrm{eV}\right.$ ) and high density ( $\mathrm{n}_{e}^{\text {div }} \geq \mathrm{n}_{e}^{\text {main }} \geq 3-10 \times 10^{19}$ $\mathrm{m}^{-3}$ ) at the divertor plates indicate that DIII-D obtains a high-recycling divertor during $\mathbf{H}$-mode. DEGAS neutral transport modeling is consistent with these results.

(4) The duration of good $\mathrm{H}$-mode confinernent is limited by Edge Localized Modes (ELMs) rather than increasing impurity radiation.

(5) The dominant impurities, $\mathrm{C}$ and $\mathrm{Ni}$, are concentrated in the edge plasma $(r / a \geq 0.5)$ for $I_{p} \geq 1 \mathrm{MA}$.

(6) Global impurity concentrations fall markedly with increasing plasma current.

The remainder of this report is outlined as follows. Section I contains a brief description of the DIII-D tokamak and the relevant diagnostics. Section II summarizes the divertor plasma parameters obtained during the $\mathrm{H}$-mode and describes the heat loads on the divirtor target plates. Section III presents the results of measurements of the central radiation and impurity content using data from an EUV spectrometer and a 21 channel bolometer array. Section IV concludes with a discussion of the implication of these results for the CIT and ITER tokamak designs.

\section{Device Configuration}

The single null, open divertor plasma configuration of DIII-D is shown in Fig. 1 , which includes a cross section of the vacuum vessel and the MHD equilibrium magnetic flux surfaces for a typical H-mode discharge. The location of the flux sirfaces was determined using the magnetic fitting code EFITD. In the usual case, the $X$-point is positioned $15-20 \mathrm{~cm}$ above the target plates and a gap of 3-5 cm between the separatrix and the main limiter is maintained by active feedback control. Below the $X$-point, the two legs of the separatrix intersect the vessel floor at an angle of about $45^{\circ}$, at which point the flux surfaces have expanded a factor of 3.5 on the outboard side and 7 at the inboard intercept relative to that at the midplane. At the target plates the angle of the actual field lines relative to the graphite tiles depends upon $q$, but is usually a shallow angle of less than $3^{\circ}$ owing to $B_{T} \gg B_{p}$.

The interior walls of DIII-D are Inconel, and $15 \mathrm{~m}^{2}$ of the $76 \mathrm{~m}^{2}$ of the wall surface are covered with graphite. Approximately one-third of the graphite is used 
for the divertor target plates, which consist of $1.4 \mathrm{~cm}$ thick slabs of POCO AXF-5Q graphite brazed to an Inconel base plate. These tiles are mounted in two concentric bands on the vessel floor, as indicated by the raised sections shown in Fig. 1. Conduction cooling to the water-cooled vessel walls keeps the bulk temperature of the divertor targets below $100^{\circ} \mathrm{C}$ during normal operation.

A broad range of divertor-region plasma diagnostics is used on DIII-D. Plasma density and electron temperature at the target plates are determined by a radial array of Langmuir probes which span the divertor region. An absolutely calibrated $\mathrm{H}_{\alpha}$ TV camera and several photodiodes with $\mathrm{H}_{\alpha}$ filters are used to measure the radial profiles of neutral hydrogen emission from the divertor plasma. Targel-plate surface temperature measurements, which are processed to yield the incident power as a function of space and time, are obtained with a calibrated IR TV camera sensitive in the 8-12 $\mu \mathrm{m}$ wavelength range. In addition, six calibrated :onization gauges measure the neutral molecular density in the divertor, near the plasma midplane, and at the top of the vessel.

\section{Plasma Conditions at the Divertor Targets}

In this section we document the plasma parameters adjacent to the divertor target plates. Plasma conditions here will determine the longevity of the divertor armor in a fusion reactor since the sputtering and erusion rates increase rapidly with increasing electron temperature, and local heat loads must be maintained at acceptable levels. Conditions at the divertor target are largely determined by the incident power flowing into the scrape-off-layer (SOL). This pover flow is itself a function of plasma transport from the core to the edge plasma near the separatrix. A complex interrelationship thus exists between the divertor plasma conditions and the global plasma parameters.

It is known that edge plasma transport is reduced significantly for H-mode discharges [3]. This is evident from measurements of the edge density and temperat ure profiles at the plasma midplane, shown in Fig. 2. These plots connect data obtained from the Thomson scattering system with that obtained by a moveable Langmuir probe. They show that the edge gradients steepen following the transition from $\mathrm{L}$ mode to $\mathrm{H}$-mode confinement. While $1.5 \mathrm{~cm}$ inside the separatrix the density and temperature are higher $\left(n_{e} \simeq 7 \times 10^{19} \mathrm{~m}^{-3}\right.$ and $\left.T_{e} \simeq 300 \mathrm{eV}\right)$ in the H-mode phase, 
outside the separatrix they are lower $\left(\mathrm{n}_{e} \simeq 5 \times 10^{18} \mathrm{~m}^{-3}\right.$ and $\mathrm{T}_{e} \simeq 50 \mathrm{eV}$ ). Thus, power flow to the divertor should be reduced during $H$-mode since $n_{\varepsilon}$ and $T_{c}$ are lower on the open field lines.

IR TV measurements [4] of the divertor heat flux are consistent with the observed changes in the SOL plasma, as in Fig. 3. At the transition from L-mode to $\mathrm{H}$-mode confinement, the peak power incident on the target plates drops by $20-50 \%$, the location of the peak heat flux shifts closer to the separatrix, and the profile width is reduced from $5-7 \mathrm{~cm}$ (1/e full width) to less than $3 \mathrm{~cm}$ at the outside intercept for $I_{p}=1.2 \mathrm{MA}, \mathrm{B}_{\mathrm{T}}=2.1 \mathrm{~T}$, and $\bar{n}_{\mathrm{e}} \simeq 5 \times 10^{19} \mathrm{~m}^{-3}$. Since both the peak value and the width of the heat flux profile are reduced in H-mode, the total integrated power deposition $\left(\int 2 \pi r P(r) d r\right)$ drops from the typical $L$-mode value of $0.3 P_{\text {inj }}$ to approximately $0.1 \mathbf{P}_{\mathrm{inj}}$.

The highest steady-state divertor heat loads are observed during NBI heating with L-modu confinement. Peak power density at the outboard intercept is typically $0.5 \mathrm{MW} / \mathrm{m}^{2}$ per MW of NBI power, but this may vary by a factor of 2 or more because of measured target plate misalignments of up to $0.3 \mathrm{~cm}$. This peak is displaced radially away from the separatrix intercept by approximately $2 \mathrm{~cm}$. Power deposition at the inboard intercept is considerably lower $\left(\leq 1 \mathrm{MW} / \mathrm{m}^{2}\right)$ and is displaced from the separatrix by 4-6 $\mathrm{c} n$, as expected from the flux expansion. Integrating over each heat deposition profile shows a factor of 2 to 3 (greater on the outboard intercept) asymmetry in the total power flow. This may be explained by preferential heat loss at the outboard midplane coupled with a longer path length for heat conduction to the inner target plates.

Simultaneous $\mathrm{H}_{\alpha}$ emission and electron temperature measurements also show asymmetries in the divertor plasma during the L-mode phase of NBI. Langmuir probe data indicates that $\mathrm{T}_{\mathrm{e}}$ can be about a factor of 2 higher at the outboard intercept than at the inside $\left(T_{e} \simeq 15-20 \mathrm{eV}\right.$ vs $\left.T_{e} \leq 12 \mathrm{eV}\right)$. On the other hand, the $H_{\alpha}$ emission is frequently brightest at the inside. It is possible to reconcile these asymmetries by assuming that the local heat flux is proportional to the local electron temperat ure and the jon flux; combining the heat flux measurements with the $\mathrm{T}_{e}$ data then gives similar ion fluxes at each strike point. However, since the ratio of $\mathrm{H}_{\alpha}$ photons/ionization increases as $T_{e}$ decreases, we expect to see more $H_{\alpha}$ at the inner separatrix. 
A more complete picture of the plasma-neutral interactions occurring at the divertor plates is provided by the DEGAS neutral transport code. Using the measured divertor-target power deposition and electron temperature to estimate the neutral source function at the plates (each $\mathrm{H}^{+}$produces $0.5 \mathrm{H}_{2}$ molecules), it calculates the expected $\mathrm{H}_{\alpha}$ emission and neutral pressures for a typical DIII-D H-mode discharge. We find that the steady-state $\left(\overline{\mathrm{n}}_{\mathrm{e}} \simeq 5 \times 10^{19} \mathrm{~m}^{-3}\right)$ can be maintained by high recycling at the divertor plates, so that 86 Torr- $1 / \mathrm{s}$ of core ionization (ionization within the separatrix) is provided by 8600 Torr-l/s of ionization at the target plates. Comparison of the calculated $\mathrm{H}_{\alpha}$ brightness in the divertor region vs the measured brightness shows agreement to within a factor of 2 . Furthermore, the predicted neutral pressures also agree with the measured values, and show that large pressure ratios $\left(P_{\text {divertor }} / P_{\text {midplane }} \simeq 10-50\right)$ are maintained in the open divertor geometry.

Having characterized the divertor plasma obtained during the quiescent $\mathrm{H}$-mode, some discussion of Edge Localized Modes (ELMs) is now appropriate. In DIIl-D two types are observed: giant ELMs, which cause a loss of plasma particles and 10-20\% of the stored kinetic energy, and small, so-called "grassy" ELMs which have little directly observable effect on the plasma. Generally, the giant ELMs occur when the NBI power level is well above $\left(P_{i n j} \geq 6 \mathrm{MW}\right.$ for $\mathrm{H}^{0}$ into $\left.\mathrm{D}^{+}\right)$the $\mathrm{H}$-mode threshold and the gap between the limiter and the separatrix is large $(\geq 3 \mathrm{~cm})$.

The effect of an ELM on the edge density and temperature profiles appears in Fig. 4, which shows them before (solid) and after (open) a giant ELM. Little change in the temperature profile is observed, but a substantial loss of edge density has occurred. Profiles obtained during an ELM show that the missing particles have been expelled to the SOL, where rapid parallel loss to the divertor region follows.

The flow of plasma to the divertor region produces increased $\mathrm{H}_{\alpha}$ emission and heat deposition on the target plates. Fig. 5 shows the heat flux at the outer target plates due to a single giant ELM. The heat pulse is very rapid, with a submillisecond rise time and an overall duration of approximately $5 \mathrm{~ms}$. Peak power densities of the order of $50 \mathrm{MW} / \mathrm{m}^{2}$ are observed, although the power is distributed on a region that is roughly twice as broad as the L-mode case [Fig. 5(b)]. Integration of such heat pulses over space and time $\left[\int d t \int 2 \pi r P(r) d r\right]$ yields total energies which are comparable to that lost from the main plasma. 


\section{Radiative Losses and Impurity Concentrations}

In tokanak plasmas, divertor operation is generally characterized by reduced impurity content and radiative loss compared with similar operation with limilers. During H-mode, however, even relatively clean divertor plasmas ( $P_{\text {rad }} \leq 0.5 P_{\text {input }}$ ) are subject to impurity buildup and increased radiative loss, and in some cases [5] this accumulation quenches the $\mathrm{H}$-mode. In contrast to the somewhat discouraging impurity accumulation seen previously at low current on other machines, in DIII-D a favorable scaling of impurity content with plasma current is observed for $\mathrm{H}$-mode discharges with $I_{p} \geq 1 \mathrm{MA}$ [6]. That is, the impurity concentration falls with increasing plasma current so that, at the highest currents $\left(I_{p}=2 M A\right)$, it is significantly lower than the L-mode concentrations.

Radiative losses in DIII-D are measured using a bolometer system which consists of an array of 21 detectors that view virtually the entire minor-radius cross section of the plasma from an outboard midplane lacation. Each channel is collimated to receive radiation from a narrow chord passieg through the plasma, as shown in Fig. 6(a), and each detector has sufficient thickness to absorb $x$-rays with energies up to $10 \mathrm{keV}$. Detajled measurements of the impurity radiation are provided by three EUV spectrometers: SPRED, an absolutely calibrated multichannel instrument from the Lawrence Livermore National Laboratory that views the plasma tangential to the magnetic axis; STRS, a multichord and multichannel instrument from the Johns Hophins University with a radial view of the plasma; and GA Technologies MVUV, a multichannel spectrometer with a radial view. At present the impurity concentrations are inferred from MIST-code calculations of the total radiated power and volume-averaged $\mathbf{Z}_{\mathrm{eff}}$.

D!II-D has operated with ohmic heating over a wide range of plasma parameters [e.g., $\left.\tilde{n}_{e}=1-10 \times 10^{19} \mathrm{~m}^{-3}, I_{p}=0.4-3.0 \mathrm{MA}, B_{T}=0.8-2.1 \mathrm{~T}\right]$. A typical surface brightness profile appears in Fig. $6(\mathrm{~b})$, which shows that the peak brightness falls on the chord that is centered near the inboard separatrix intercept. Since the radiation from the region near the divertor plates is poloidally asymmetric, only data from the chords in the upper one-half of the vessel are inverted to obtain the radiation density profile within the closed flux surfaces. Over a large ensemble of ohmic divertor shots, the radiation density profiles are characteristically flat to somewhat hollow. The 
fraction of total radiated power to ohmic input power varies from 0.25 to 0.80 , with the highest value corresponding to high density operation $\left(\bar{n}_{e} \simeq 4.4 \times 10^{19} \mathrm{~m}^{-3}\right)$.

Most of the impurity radiation in ohmic plasmas is the result of $\mathrm{C}$ and $\mathrm{Ni}$ contamination. Very little oxygen is observed, and its line emission peaks during the initial "burn-through" phase of the discharge. In sharp contrast with the initial divertor results that showed $\mathrm{Z}_{\text {eff }}^{\text {lim }} \simeq \mathrm{Z}_{\text {eff }}^{\text {div }} \simeq 5-6$, present values of $Z_{\text {eff }}$ are in the range 1.5 to 2 . This cleanup follows the repair of various vacuum leaks.

Radiative losses increase with the addition of NBI heating. Plasmas with Lmode confinement exhibit gross features of the radiated power flux distribution that are very similar to those of ohmic plasmas. Here again the peak surface brightness is coincident with the inner leg of the divertor. However, inversion of the bolometer data from the upper chords yields a radiation density profile which is slightly peaked on axis. Spectroscopic measurements show that while metallic impurities (mainly $\mathrm{Ni}$ ) account for more than $50 \%$ of the radiative loss, the measured $Z_{e f f} \leq 2.0$ is due largely to carbon. These impurities apparently do not accumulate during the $\mathbf{L}$-mode phase since the total radiated power remains a constant fraction of the input power after the core plasma density and temperature reach steady-state values. For the L-mode, ,radiative losses account for approximately 30-40\% of the NBI power, with flow to the divertor target plates accounting for another 30\%; the remaining $30-40 \%$ of the losses are not measured.

Impurity behavior during $\mathrm{H}$-mode operation in DIII-D is complicated by giant ELMs, which periodically cause a loss of more than $30 \%$ of the plasma density at large radii ( $r / a \geq 0.5$ ), as can be seen in Fig. 4. These events modulate the brightness of various impurity emission lines as well as the total radiated power. Typical data from a 2.0 MA H-mode discharge with 9.5 MW of NBI power appears in Fig. 7, which shows the temporal evolution of the plasma density, edge $\mathrm{H}_{\alpha}$ emission, the brightness of two emission lines of nickel, and the total radiated power. The effect of the H-mode transition ard the ELMs is clearly seen.

At the transition from L-mode to $\mathrm{H}$-mode confinement, the radiative loss drops rapidly back to the ohmic level (data averaged over $16 \mathrm{~ms}$ ). This is largely the result of a reduction in emission from the divertor region, which follows the drop in power flow to the divertor target plates recorded by the IR TV camera. After this initial 
drop, the total radiation begins to rise monotonically, only now the profile becomes more and more hollow. Inversion of the data shows a steady decrease in radiation from the divertor region while the power radiated from the outer third of the main plasma increases steadily. Radiation from the core plasma rises more slowly. Just before the first giant ELM occurs, the total radiated power is about $40 \%$ of the input power and $Z_{\text {eff }} \simeq 1.7$.

The rising impurity radiation observed during the quicscent $\mathrm{H}$-mode has been modeled using an equilibrium solution of the MIST code. Inputs to the code inclucted the measured electron temperature and density profiles and a radial diffusion coefficient for impurities of $1 \times 10^{4} \mathrm{~cm}^{2} / \mathrm{s}$. Typical results appear in Fig. 8, which shows a romparison of the calculated radiative loss profiles due to carbon and to nickel with the inverted bolometer profile for a 1.2 MA discharge. Good agreement is obtained.

The picture which emerges from the modeling is that the impurity transport is a strong function of the plasma current during the quiescent period between ELMs. At low current ( $\mathrm{Ip} \simeq 0.8 \mathrm{MA}$ ), the total electron density and the impurity density profiles are flat and $Z_{\text {eff }}$ is relatively high $(\simeq 2.5)$. At higher plasma current both profiles become more hollow and $Z_{\text {eff }}$ falls as the impurities become more concentrated in the edge plasma. This trend continues up to at least $I_{p}=2.0 \mathrm{MA}$, where $Z_{\text {ef }} \simeq 1.7$. The spectroscopic data are consistent with this picture, as indicated by the data of Fig. 7. It is found that between ELMs, the brightness of the $165 \AA$ line of Ni XVIII (ionization potential of $600 \mathrm{eV}$ ) increases linearly, while that of the $118 \AA \mathrm{Ni} X X \mathrm{~V}$ line (ionjzation potential of $2300 \mathrm{eV}$ ) oscillates about an equilibrium value. While the ELM-produced modulation of the Ni XVIII is observed at all values of plasma current, the steady-state brightness of the $\mathrm{Ni} X X V$ line drops with increasing plasma current even though the central electron density rises.

\section{Discussion}

Operating experience with the single-null open divertor configuration in DHI-D is relevant to planning for next generation tokamak devices such as CIT and ITER. The data from $\mathrm{H}$-mode discharges have implications both for divertor design and for obtaining optimum plasma conditions for ignition. For example, since DIII-D has demonstrated that it is possible to obtain good $\mathrm{H}$-mode confinement ( $\tau_{\mathrm{E}} \geq 2 \tau_{\mathrm{E}}^{\mathrm{L}}$-mode) with a high recycling (low $T_{e}$ and high $n_{e}$ ) open divertor, there is little motivation 
to design a new device with a highly baffled divertor and its accompanying restrictions on possible plasma shapes. The neutral pressure measurements support this conclusion since the divertor/midplane pressure ratio is already large.

Our measurements of the divertor-target heat loads have led us to conclude that the best way to minimize the target heating is to modulate the strike points by sweeping the $\mathrm{X}$-point radial position during the shot. This is preferable to designing highly shaped target plates since we find what the location of the peak heat flux can vary considerably during a shot. The peak moves closer to the separatrix at the onset of NBI and again at the $\mathrm{H}$-mode transition, and its width varies with both NBI power and plasma density. With the onset of so-called locked modes [7], the heat deposition profile has been observed to bifurcate into two peaks situated outside the separatrix intercepts [8]. And during ELMs, when the divertor-target power flux is up to ten times the L-mode value, the energy is deposited over much wider regions. Thus, a dynamic $\mathbf{X}$-point position seems the best way to provide both flexibility in plasma shaping and protection of the target plates themselves.

The choice of graphite for the divertor target plates is motivated by the desire to mir $i$.jize the impurity influx produced by sputtering. Spectroscopic measurements in JT-60 show much lower impurity levels when using graphite target plates rather than $T: C$ coated molybdenum target plates, although the total radiated power was not limiting plasma operation in either case. In DIII-D, radiative collapse of the $\mathrm{H}$-mode is not observed as it is in both ASDEX (metal targets) and JET (graphite targets), but the radiative losses are increasing throughout the quiescent $\mathrm{H}$-mode phase. The most important factor in controlling the impurity influx is to maintain a low electron temperature at the target plates so that the ion impact energy ( $\simeq 3 T_{e}$ ) is below the threshold for sputtering. A related issue, which still needs resolving, is that of the effect of the apparent inboard/outboard asymmetry in the electron temperature at the target plates. This asymmetry may produce norsuniform surface erosion with subsequent material migration within the divertor region.

The lower radiative losses observed in DIII-D are also a consequence of operating at high plasma currents. As shown above, a favorable scaling of impurity content with plasma current is obtained. We believe that this is a result of the edge-peaking of the density profile observed with increasing plasma current, so that impurities are confined to the edge plasma. This not only directly reduces the core impurity content, 
but allows ELMs, which produce a loss of the edge plasma, to more efficjently remove impurities from within the closed flux surfaces to the open field lines in the scrapeoff-]ayer. This suggests that an optimal $\mathrm{H}$-mode plasma with rainimal radiative losses may be one in which the density profile is slightly hollow and which has some low level of ELM activity to serve as an impurity pumping mechanism.

\section{Acknowledgments}

The DIII-D boundary physics program at GA Technologies Inc. enjoys the active support of participants from Lawrence Livermore National Laboratory (LLNL); Japan Atomic Energy Research Institute (JAERI); Johns Hopkins University (HOP. KINSU); Princeton Plasma Physics Laboratory (PPPL); University of California, Los Angeles (UCLA); and Max-Planck-Institut für Plasmaphysik, Garching (JAR). This work was performed under the auspices of the U.S. Department of Energy by Lawrence Livermore National Laboratory under contract $W-7405-$ Eng-48 and by GA Technologies Inc. under contract DE-ACO3-84ER51044.

The data presented in this document have been presented previously as a GA Technologies Report GA-A19101. 


\section{REFERENCES}

1. K.H. Burrell et al., Phys. Rev. Lett. 59, 1432 (1987).

2. J. Luxon et al., Plasma Phys. Cont. Nucl. Fus. Research 1986 (Proc. 11 Int. Conf. Kyoto, Japan, 1986), Vol. 1, IAEA, Vienna (1987) 1711.

3. F. Wayner et al., Phys. Rev. Lett. 53, 1453 (1984).

4. D.N. Hill et al, submitted to Nucl. Fusjon.

5. E.R. Müller et al., Max-Planck-Institut für Plasmaphysik Report IPP III/112, 1986.

6. N.H. Brooks et al., in preparation for Phys. Rev. Lett.

7. A. Kellman et al., Bull. Am. Phys. Soc. 32, 1846 (1987).

8. D.N. Hill et al., Bull. Ám. Phys. Soc. 32,1898 (1987). 


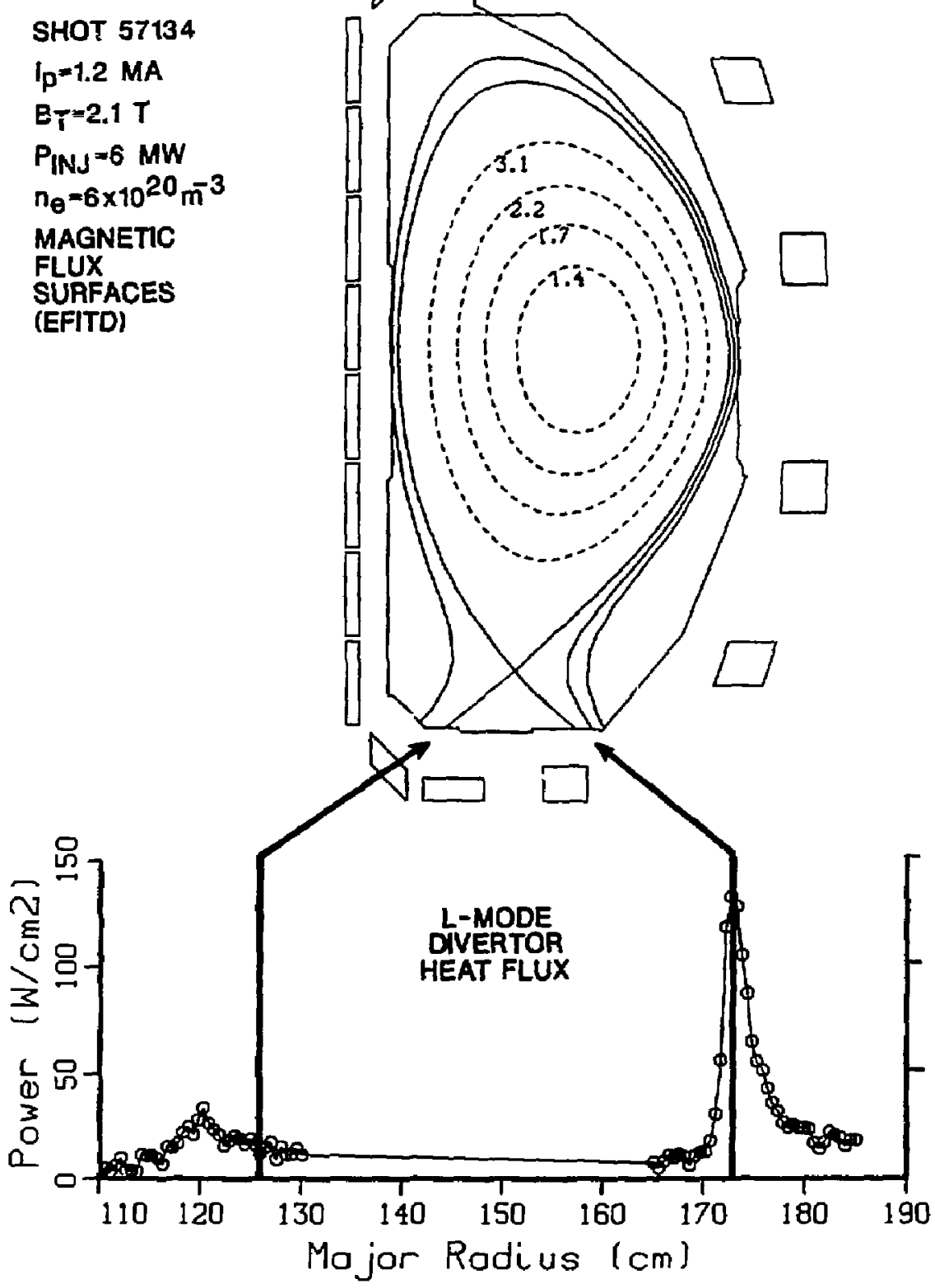

Fig. 1. Cross section of the DIII-D vessel showing magnetic flux surfaces and measured heat-flux distribution on the divertor-target plates. 

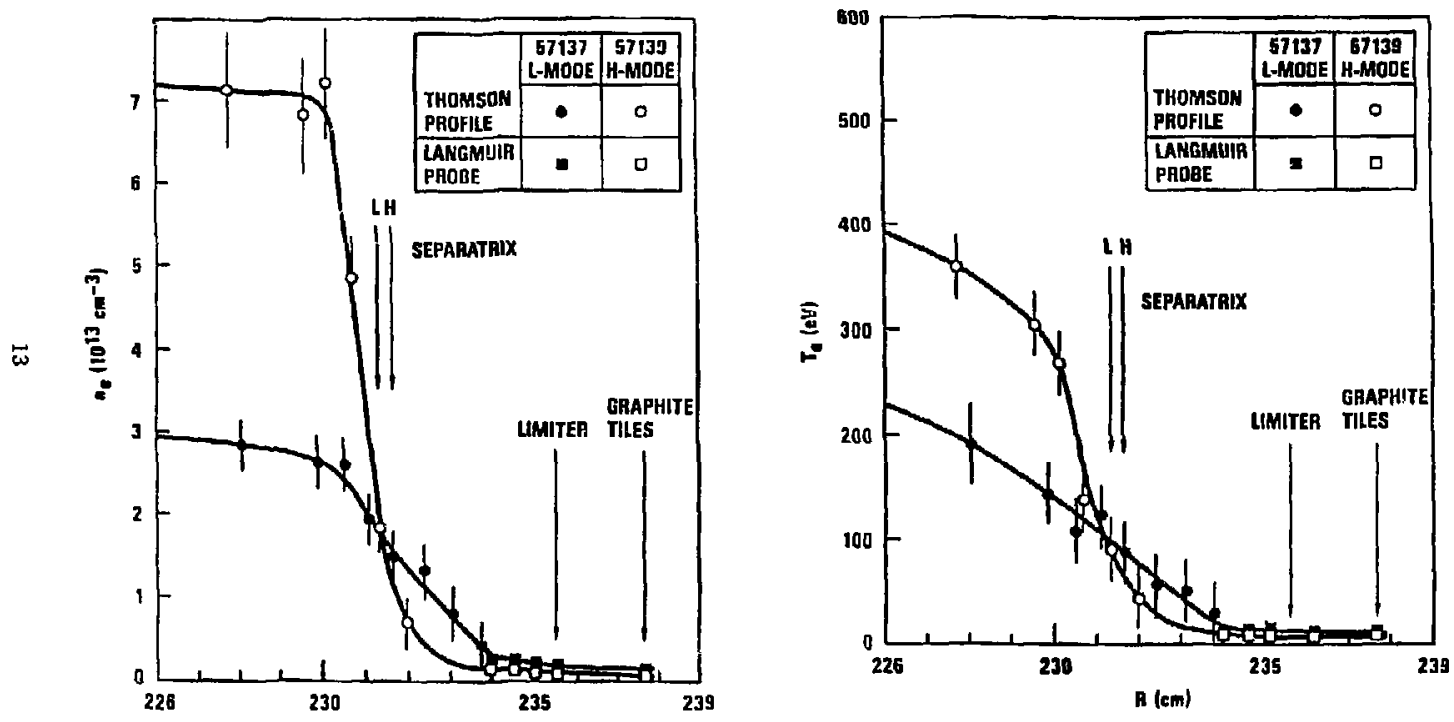

Fig. 2. Measured electron temperature and density profiles at the plasma midplane. 


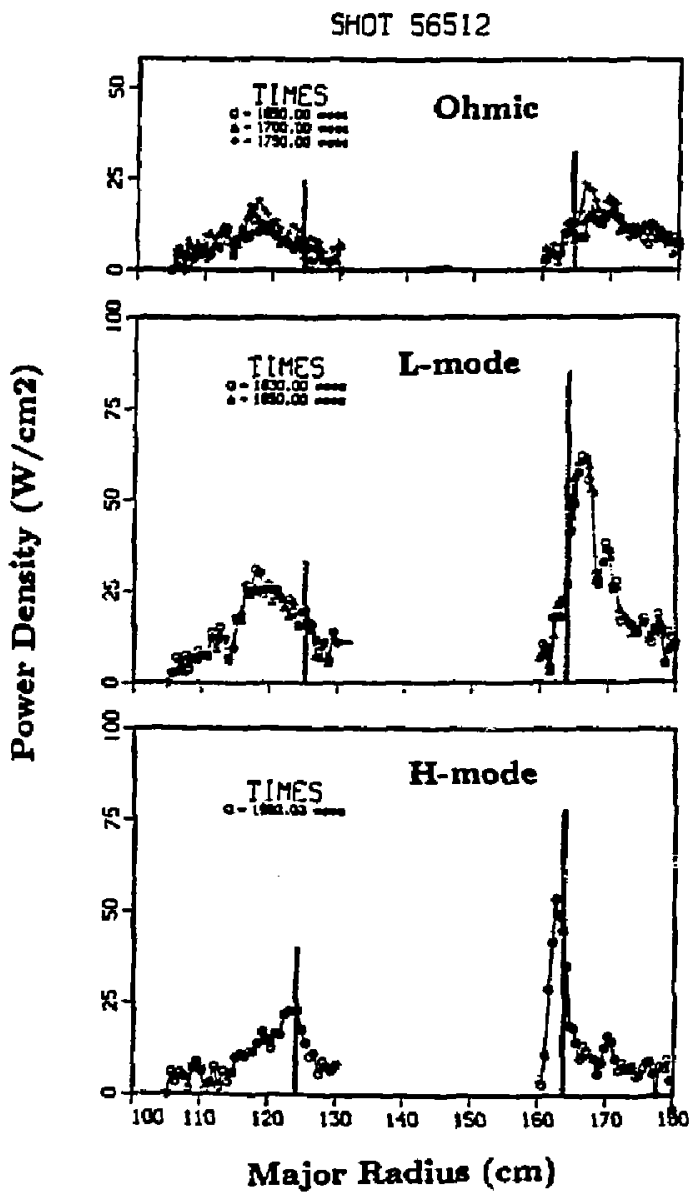

Fig. 3. Measured divertor-target power deposition profiles. 


\section{EFFECT OF ELMS ON THOMSON PROFILES}
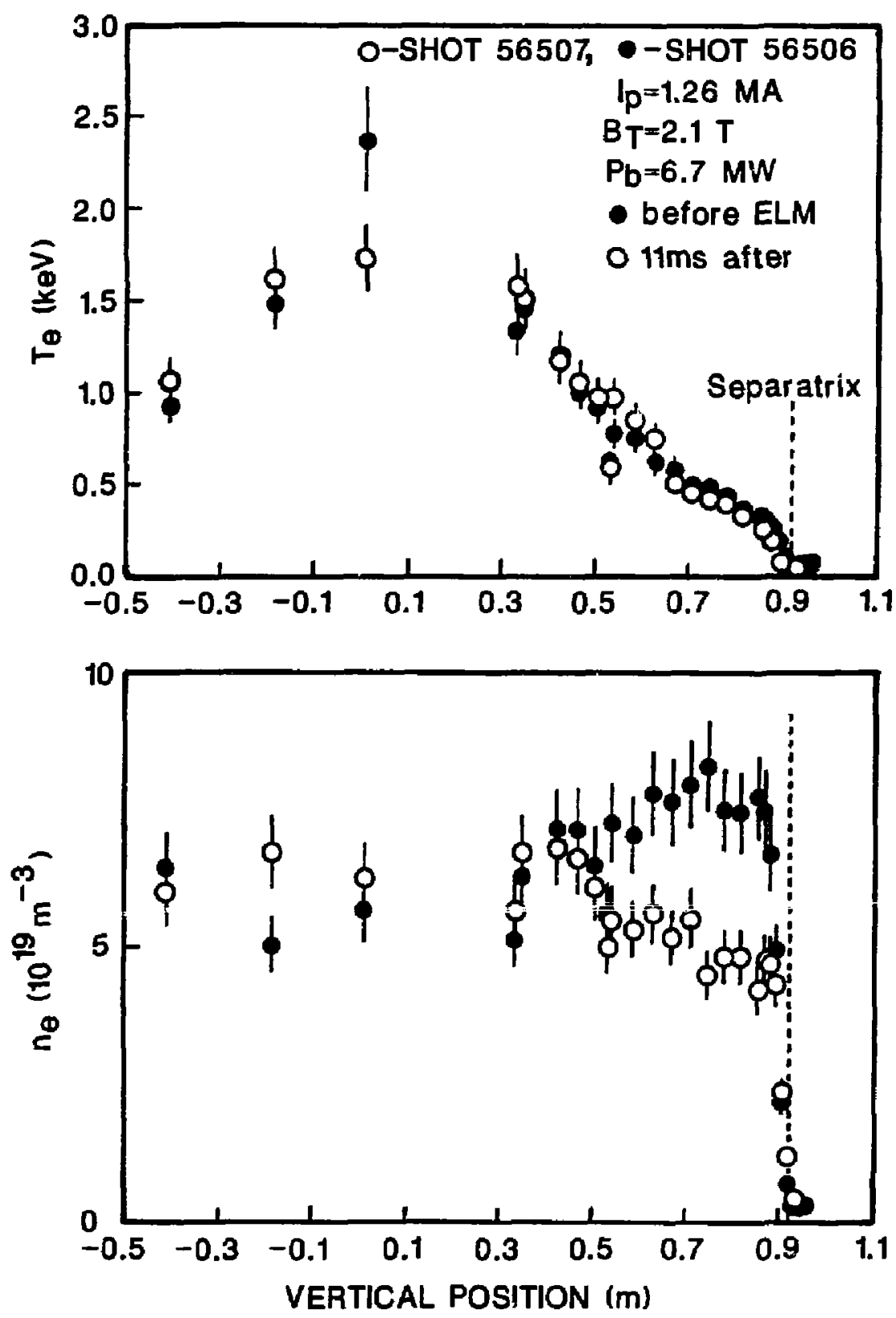

Fig. 4. The effect of a giant ELM on the edge electron density and temperature. 

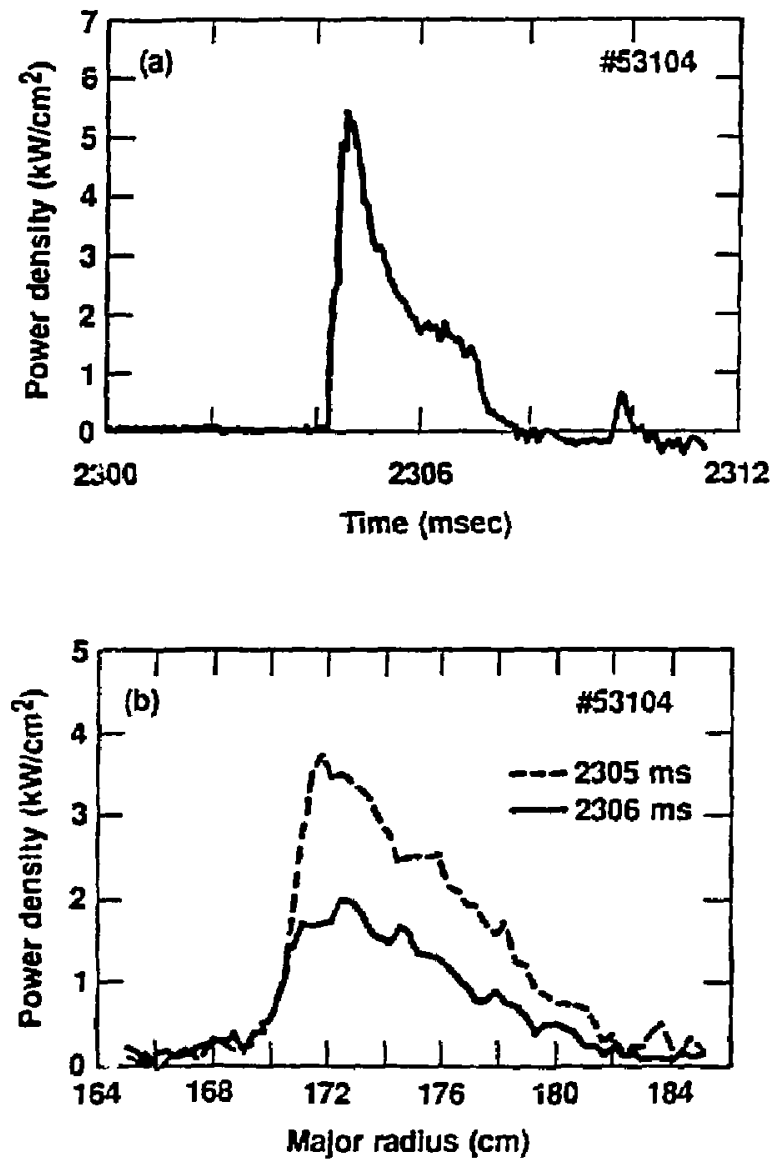

Fig. 5. Heat flix at the outer divertor-target plates due to a giant ELM: (a) Peak power density vs time; (b) Power density profile at the times indicated. 


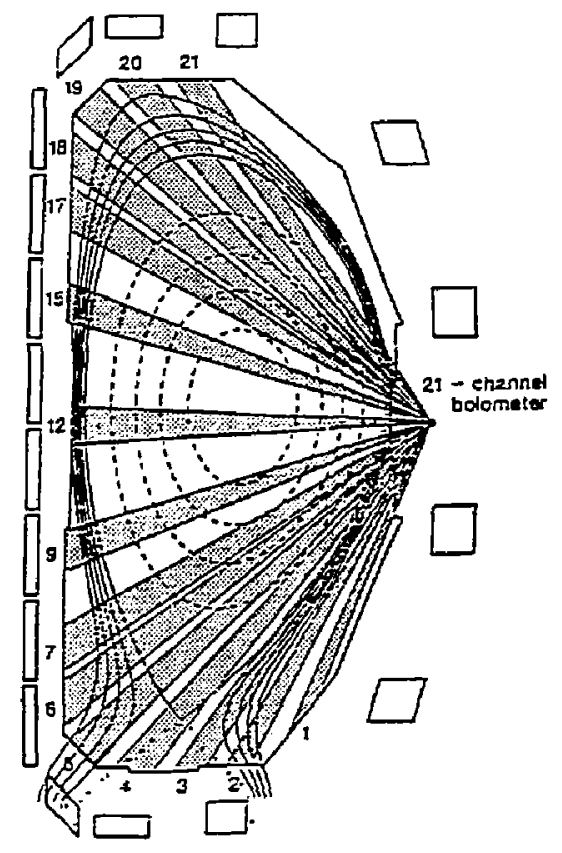

(a)

\section{SURFACE RADIATION DISTRHBUTION}

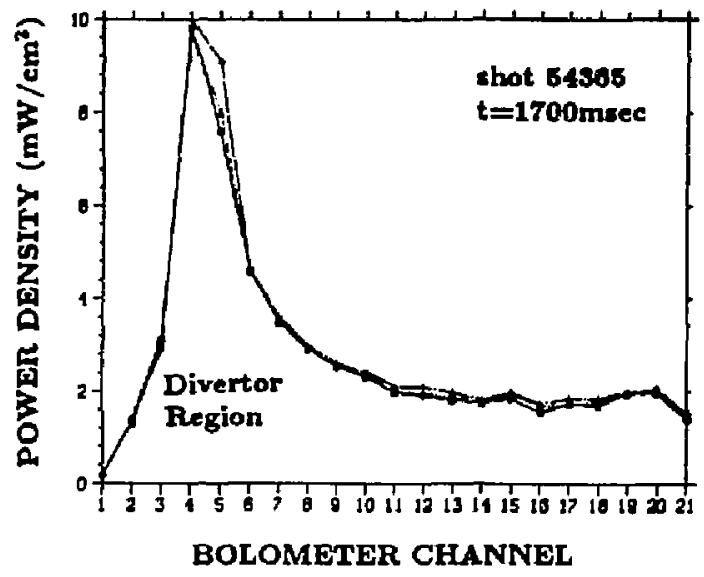

(b)

Fig. 6. Measurements of the total radiated power: (a) Field-of-view of the 21 channel bolometer array; (b) Typical surface brightness profile for an ohmically heated plasma. 

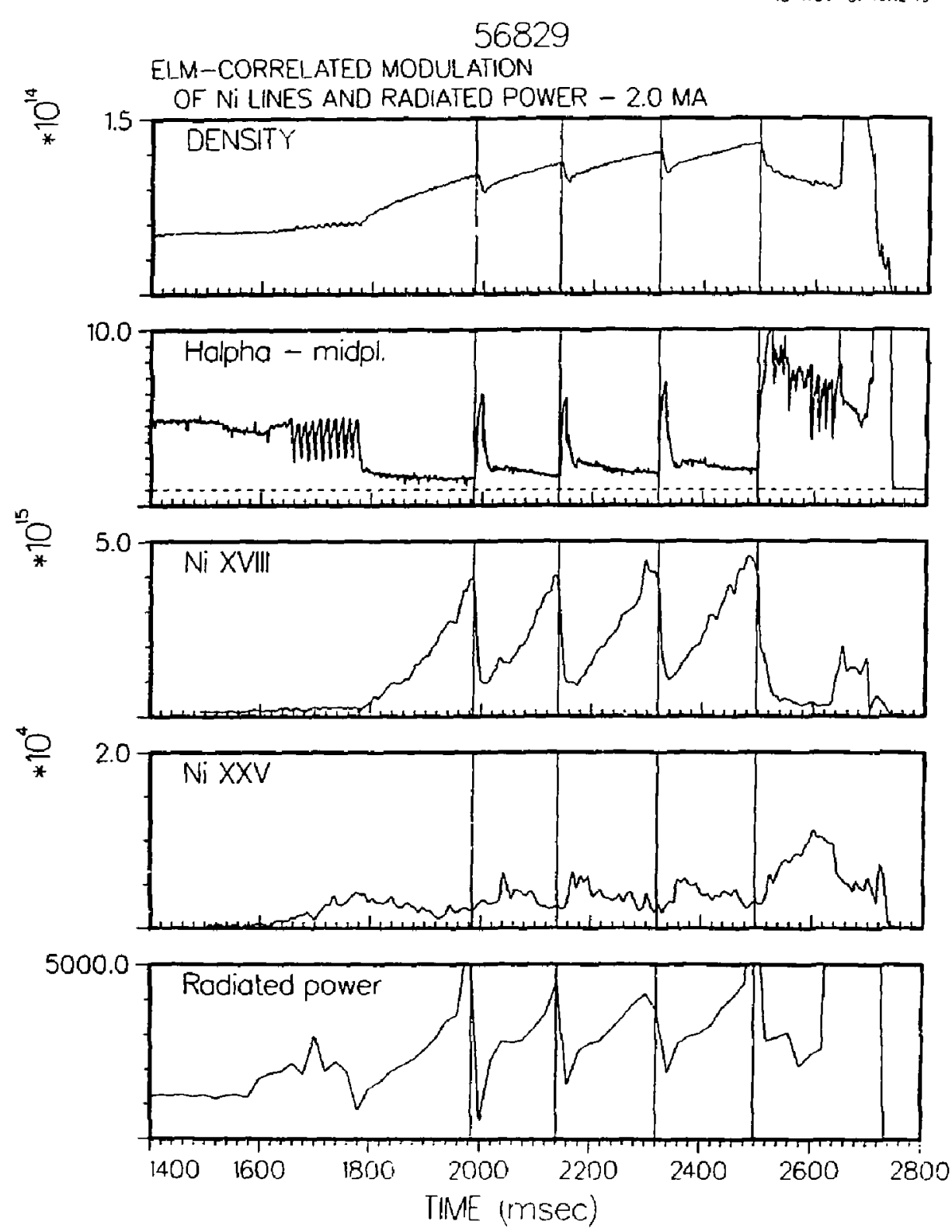

Fig. 7. Typical data from a high current $\mathrm{H}$-mode discharge showing the temporal behavior of the (top to bottom) plasma density, midplane $H_{\alpha}$ brightness, Ni XVIII brightness at $165 \AA$, Ni XXV brightness at $118 \AA$, and the total radiated power. 
RADIAL PROFILES OF RADIATIVE LOSS

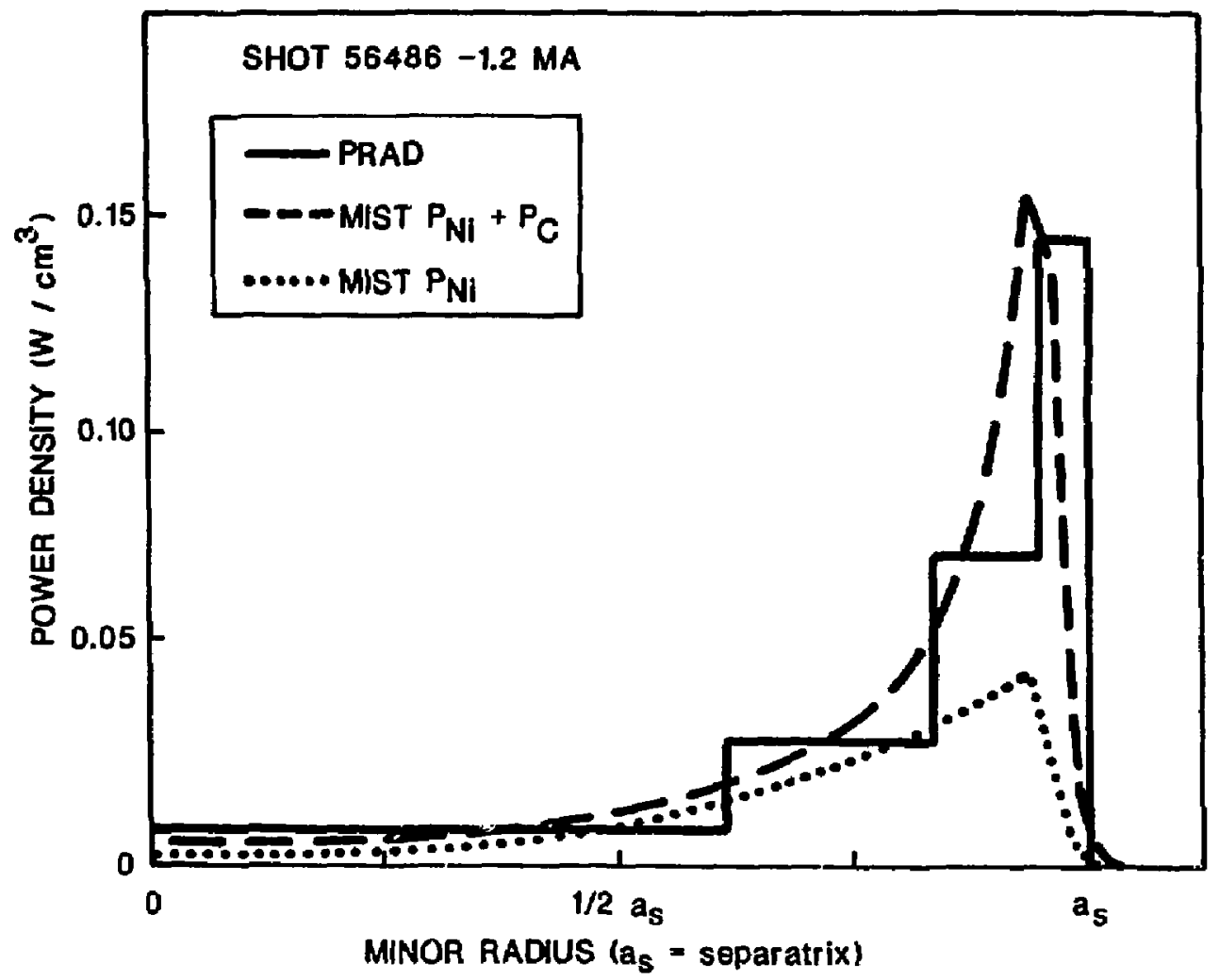

Fig. 8. Comparison of MIST-code calculations of impurity radiation profiles with an inversion of the bolometer profile of total radiation. 